\title{
Anaplasmataceae subversion of the lysosomal activity
}

\begin{abstract}
Pathogenic bacteria belonging to the family Anaplasmataceae include species of the genera Ehrlichia, Anaplasma and Neorickettsia. These bacterial obligate intracellular parasites have evolved diverse mechanisms for evasion of host cellular defenses. One of these mechanisms involves adaptations for survival in distinct intracellular compartments that allow their replication in seclusion from lysosomal killing. Here, I review the intracellular niches inhabited by these obligate intracellular parasites such as: arrested early endosomes, lysosomes, and vesicles that do not fuse with the endosomal compartment but intersect with an exocytic pathway.
\end{abstract}

Keywords: Anaplasmataceae, intracellular niches, lysossomal evasion, cell markers
Volume 6 Issue I - 2018

\section{Rosiane Nascimento Alves}

Biomedical Sciences Institute, Federal University of Uberlandia, Brazil

Correspondence: Rosiane Nascimento Alves, Instituto de Ciências Biomédicas, Universidade Federal de Uberlândia, Av. Pará, 1720, Umuarama, 38400-902, Uberlândia-MG, Brazil, Tel +55 (34) 3225848I, Email rosiane.alves@ufu.br

Received: August 0I, 2017 | Published: January 22, 2018

\section{The different members of the anaplasmataceae family}

Obligate intracellular bacteria with unique host cell specificities, such as members of the family Anaplasmataceae, have developed several mechanisms to ensure immune evasion of host cellular defences. These mechanisms involve adaptations for survival and replication within nonlysosomal 4. intracellular vacuoles which are nothing more than host cell membrane-bound inclusions called morulae. ${ }^{1}$ This is particularly important for these bacteria because they exclusively reside in professional phagocytes that have as their main function the destruction of engulfed bacteria through lysosomal degradation. ${ }^{2,3}$

The better known bacteria whose cytoplasmic inclusions do not fuse with lysosomes and which are currently included in this family are Ehrlichia spp, Anaplasma spp. and Neorickettsia spp..$^{4-6}$ Members of the genus Ehrlichia are increasingly being recognized as pathogens of human disease in the United States and other parts of the world. Two emerging infectious diseases, human monocytic ehrlichiosis (HME) caused by Ehrlichia chaffeensis and human granulocytic ehrlichiosis (HGE) caused by Anaplasma phagocytophilum (formerly E. equi and HGE agent), have only been recognized over the last few years. ${ }^{1,7}$ Beyond that, the global canine pathogen Ehrlichia canis has been isolated from a human in Venezuela, and several patients with clinical signs similar to HME were found to be infected with $E$. canis at the same country.

\section{Intracellular niches and their cellular markers}

Caveolae- or lipid raft-mediated endocytosis is a vesicle trafficking system that bypasses phagolysosomal pathways, and is thus utilized by a wide variety of pathogenic microorganisms to invade host cells. ${ }^{10}$ The entry and intracellular infection of E. chaffeensis and $A$. phagocytophilum involve cholesterol-rich lipid rafts or caveolae and glycosylphosphatidylinositol (GPI)-anchored proteins. ${ }^{11}$ Moreover $E$. chaffeensis and A. phagocytophilum inclusions were not colocalized with CD63 or LAMP-1 (lysosome-associated membrane protein-1), lysosomes membrane glycoproteins, wich can be used as markers of lysosomal fusion. The absence of these lysosomal markers on ehrlichial inclusions indicates that ehrlichial inclusions do not fuse with lysosomes. ${ }^{4,5,12,13}$ Nevertheless, these bacteria use different strategies to avoid lysosomal fusion and create their safe havens. When a human promyelocytic leukemia cell line HL-60 is coinfected with $E$. chaffeensis and A. phagocytophilum, they resided in separate inclusion compartments with different characteristics within the same cell. $^{5}$

Cytoplasmic inclusions containing E. chaffeensis have characteristics of early endosomes, presenting the markers Rab5, early endosome antigen 1 (EEA1) and transferrin receptor (TfR). ${ }^{4,5}$ Furthermore, minimal accumulation of the acidotropic base 3-(2,4-dinitroanilino)-3'-amino-N-methyldipropylamine (DAMP) and the vacuolar $\mathrm{H}+$ ATPase within $E$. chaffeensis morulae suggested that the vesicle is only weakly acidic. ${ }^{2,4} E$. chaffeensis thus appear to block maturation of endosomes and remain in an early endosomal compartment, thereby avoiding lysosomal fusion. In contrast, the inclusion compartment of $A$. phagocytophilum do not possess these early endosome characteristics. ${ }^{5,13}$ Therefore, vacuoles containing these bacteria incorporated endocytosed colloidal gold particles and were labeled to the cation-dependent mannose-6-phosphate receptor (M6PR). The M6PR is involved with delivery of lysosomal enzymes to late endosomes and lysosomes and recycles from the Golgi apparatus to endosomal compartments and back again. ${ }^{13}$ Therefore, A. phagocytophilum resides in compartments belonging to endocytic pathway.

The cytochemical staining for acid phosphatase marks late endosomes and lysosomes, as well as vacuoles that merge with these organelles. ${ }^{1}$ Previous studies indicated that the vacuole that contains $N$. risticii or A. phagocytophilum showed no labelling for acid phosphatase activity in cells not treated with the antibiotic oxytetracycline. Once treated, the cells showed a significant increase in the co-localisation of lysosomal markers and vacuoles that contains the bacteria, suggesting that this drug affect the ability of these bacteria to inhibit lysosomal fusion. ${ }^{6}, 14$ Therefore inhibition of ehrlichial protein synthesis by oxytetracycline causes a failure to inhibit the maturation of endosomes to lysosomes, with resultant destruction of the parasites. Similarly, Alves et al., ${ }^{15}$ demonstrated that intact cytoplasmic inclusions of E. canis are rarely labelled with acid phosphatase compared to deteriorated inclusions suggesting that the spreading process of $E$. canis in vitro is dependent on lysosomal evasion. These data indicate that inactive or dead intracellular microorganisms lose their ability to inhibit phagosome-lysosome fusion. Another study showed that lysosomal proteins such as cathepsin D, cathepsin S, and lysosomal acid phosphatase were not detected in E. chaffeensis phagosome preparations by proteomics methods. ${ }^{2}$ Moreover, the inhibition of lysosomal fusion is specific to parasitophorous vacuoles, 
as intracellular $N$. risticii or A. phagocytophilum or E. chaffeensis do not inhibit lysosomal fusion with phagosomes containing latex particules ingested by the same cell. , $, 6,14^{2}$

Despite the above mentioned studies demonstrated that Ehrlichiacontaining vacuole (ECV) does not fuse with lysosomes, an essential condition for Ehrlichia to survive inside phagocytes, the mechanism of inhibiting the fusion of the phagosome with lysosomes is not clear. Thus, Cheng et al., ${ }^{2}$ detected Rab7, a late endosomal marker, in E. chaffeensis phagosomes by proteomic and immunofluorescene analysis. Beyond that these phagosomes were acidified at approximately $\mathrm{pH} 5.2$, suggesting that the E. chaffeensis vacuole was a late endosome. Thereby, E. chaffeensis vacuoles were capable of fusing with early endosomes and maturing into late endosomes, without lysosome fusion. This phenomenom by which E. chaffeensis inhibits phagosome-lysosome fusion is to modify its vacuolar membrane composition, rather than by regulates the expression of host genes involved in trafficking.

\section{Conclusion}

Despite being based on different strategies according to the member of the family Anaplasmataceae, the evasion of lysosomal fusion by ehrlichial inclusions is fundamental to the survival and replication of this pathogen. Additional analyses of the ECV molecular composition could decipher the mechanism by which Ehrlichia inhibits phagosomelysosome fusion in the host cell and may facilitate the development of new therapeutic strategies.

\section{Acknowledgements}

None.

\section{Conflict of interest}

The author declares no conflict of interest.

\section{References}

1. Hackstadt T. The diverse habitats of obligate intracellular parasites. Curr Opin Microbiol. 1998;1(1):82-87.

2. Cheng Y, Liu Y, Wu B, et al. Proteomic analysis of the Ehrlichia chaffeensis phagosome in cultured DH82 cells. PLoS One. 2014;9(2):e88461.
3. Rikihisa Y. Ehrlichia subversion of host innate responses. Curr Opin Microbiol. 2006;9(1):95-101.

4. Barnewall RE, Rikihisa Y, Lee EH. Ehrlichia chaffeensis are early enodosomes which selectively accumulate transferrin receptor. Infect Immun. 1997;65(4):1455-1461.

5. Mott J, Barnewall RE, Rikihisa Y. Human granulocytic ehrlichiosis agent and Ehrlichia chaffeensis reside in different cytoplasmic compartments in HL-60 cells. Infect Immun. 1999;67(3):1368-1378.

6. Wells MY, Rikihisa Y. Lack of lysosomal fusion with phagosomes containing Ehrlichia risticii in P388D1 cells: abrogation of inhibition with oxytetracycline. Infect Immun. 1988;56(12):3209-3215.

7. Rikihisa Y. Mechanisms to create safe haven by members of the family of Anaplasmataceae. Ann N Y Acad Sci. 2003;990:548-555.

8. Perez M, Rikihisa Y, Wen B. Ehrlichia canis-like agent isolated from a man in Venezuela: antigenic and genetic characterization. J Clin Microbiol. 1996;34(9):2133-2139.

9. Perez M, Bodor M, Zhang C, et al. Human infection with Ehrlichia canis accompanied by clinical signs in Venezuela. Ann N Y Acad Sci. 2006;1078:110-117.

10. Lafont F, van der Goot FG. Bacterial invasion via lipid rafts. Cell Microbiol. 2005;7(5):613-620.

11. Lin M, Rikihisa Y. Obligatory intracellular parasitism by Ehrlichia chaffeensis and Anaplasma phagocytophilum involves caveolae and glycosylphosphatidylinositol-anchored proteins. Cell Microbiol. 2003;5(11):809-820.

12. Chen JW, Chen GL, D'Souza MP, et al. Lysosomal membrane glycoproteins: properties of LAMP-1 and LAMP-2. Biochem Soc Symp. 1986;51:97-112.

13. Webster P, IJdo JW, Chicoine LM, et al. The agent of Human Granulocytic Ehrlichiosis resides in na endosomal compartment. J Clin Invest. 1998;101(9):1932-1941.

14. Gokce HI, Ross G, Woldehiwet Z. Inhibition of phagosome-lysosome fusion in ovine polymorphonuclear leucocytes by Ehrlichia (Cytoecetes) phagocytophila. J Comp Pathol. 1999;120(4):369-381.

15. Alves RN, Levenhagen MA, Levenhagen MM, et al. The spreading process of Ehrlichia canis in macrophages is dependent on actin cytoskeleton, calcium and iron influx and lysosomal evasion. Vet Microbiol. 2014;168(2-4):442-446. 